\title{
LE MUSÉE DE NICHE. NOUVEL EXPLOIT DANS LA MUSÉOGRAPHIE
}

\section{THE SMALL MUSEUM. NEW EXPLOITATION IN MUSEOGRAPHY}

\author{
Mădălina IACOB \\ Universitatea de Vest, Timişoara/ West University of Timişoara \\ e-mail: madalina.iacob@e-uvt.ro
}

\begin{abstract}
In all the complexity of the museum study, there is a slight border that deserves all the attention of the researchers: the one of the niche museums. This work starts from the idea according to which the museum becomes a symbol of cultural practice in the contemporary era. In addition to the successful museums that are being built and built in the city, there is a new tendency to transform some spaces into small museums. These, in full process of heritage building, can highlight a series of features and characteristics of a society. The research of the niche museum starts from Ulf Hannerz, who says in his study that anthropology must renew its limits, it must take into account urban life. Researchers should not focus only on rural areas, in small, homogeneous communities, especially as they are outside Western societies Urban anthropology must be based on a range of social and cultural phenomena that will rarely be found in rural areas and which must be analyzed in the light of the diversity of human societies in general, says Ulf Hannerz, like the diversity of museums. From the chocolate museum, the lace museum, the cake museum, the cheese museum or the flower museum, all these culturally-rendered spaces are meant to anonymously remove some objects or crafts that are characteristic of a particular group and which subsequently become part of the immaterial cultural heritage. The Dictionary of Ethnology and Anthropology defines the study of anthropology regarding museography as a necessity inherent in the advancement of ethnography. Researchers such as Robert Park, Ulf Hannerz, Clifford Geertz, André Malraux or Chiara Bortolotto have studied the relationship of the museum with the city, thus implicitly with society. The conclusions they draw have the following aspect in common: the museum has the intrinsic ability to model and structure the immediate society.
\end{abstract}

Keywords: musée; anthropologie; patrimoine; art; études culturelles.

Le dictionnaire d'Ethnologie et d'Anthropologie définit l'étude de l'anthropologie concernant la muséographie comme une nécessité inhérente à l'essor de l'ethnographie. Les chercheurs comme Robert Park, Ulf Hannerz, Clifford Geertz, André Malraux ou Chiara Bortolotto ont étudié la relation du 
musée avec la ville, et implicitement avec la société. Les conclusions tracées en commun par ceux-ci ont l'aspect suivant : le musée a la capacité intrinsèque de modeler et de structurer la société immédiate. Selon Pierre Bourdieu, à part cette fonction il accomplit aussi la fonction économique.

L'objectif principal de cet article est de traiter le sujet de la muséographie dans l'espace urbain, parce que l'on crée un nouveau type de musée, donc un nouveau type de pratique symbolique. Positionnés souvent au cœur des villes, ces musées jouissent d'une popularité restreinte même s'ils réunissent des gens ayant en commun une passion ou un désir de connaitre. Très connus dans les espaces de la muséographie italienne et de celle française, ils ont une popularité de plus en plus élevée au sein des visiteurs. Tout musée a une histoire à raconter, qui doit refléter un morceau de l'histoire, l'histoire immédiate, l'histoire ancienne, l'histoire contemporaine de l'art en général, mais aussi de la culture. Le musée a la capacité extraordinaire de parler par sa simple présence de la communauté où il se trouve, mais aussi d'une civilisation différente et de produire des émotions. Ce bagage émotionnel est la preuve même de l'existence du patrimoine, disait la chercheuse Nathalie Heinrich.

Ce type de musée n'est pas non plus nouveau du point de vue historique. A partir même de 1990, le curateur du Musée d'Art Moderne de New York traitait le problème de ce type de musées. En outre, beaucoup de musées d'Europe peuvent être introduits dans cette catégorie. Il s'agit des musées que nous associons souvent à la communauté dont ils font partie (Kent, 1913: 1048), communauté qui participe d'une manière active au processus de patrimonialisation ${ }^{1}$ du musée, les objets prenant de la valeur par la vision de ladite communauté, de la société qui rend possible cette action. Dans la littérature roumaine de spécialité il n'y a pas d'études concernant les musées de niche.

Le musée de niche (le musée de petites dimensions) est un musée aussi petit qu'important pour certains. Ça dépend à qui l'on pose la question. Pour l'anthropologie, le musée est défini comme l'espace de l'exposition due à la patrimonialisation. Cette action cache derrière elle l'histoire d'une communauté ou d'un groupe. Le musée de niche inclut des sujets qui touchent en premier la communauté locale et ensuite celle nationale. Le musée de niche s'ouvre par des espaces privés, nés d'une passion, d'une personne ou d'un groupe pour y exposer des objets avec une valeur intrinsèque plus grande et plus précieuse que ce que l'on voit à première vue.

\footnotetext{
${ }^{1}$ Terme proposé par Pascal Dibie. «Processus par lequel les communautés humaines cherchent à préserver le passé avant de le mettre dans une collection, donc en évidence» (Dibie, 2006 :101 cité par Heinich, 2009 : 19).
} 
La bibliographie britannique et celle française incluent quelques études (de petites dimensions) à ce sujet, études qui assimilent le musée de niche au musée de petites dimensions. L'idée qui en découle est que petit ne renvoie pas à la dimension. Premièrement, il s'agit de la découpe d'un thème récurrent; spécifique, de niche. Tel un musée national (traditionnel), le musée de petites dimensions est aussi confronté à une série de problèmes, en commençant avec celui législatif.

Pour les visiteurs qui ne font pas partie d'un groupe qui montre un réel intérêt pour un tel musé et qui sont des consommateurs simples, ces musées deviennent des consommations culturelles. Pierre Bourdieu affirme que l'histoire de l'espace social

" détermine les goûts par le biais des propriétés inscrites dans une position et, surtout, par les contraintes sociales associées à certaines conditions matérielles d'existence et à un certain niveau dans la structure sociale » (Bourdieu, 2007 : 338).

Par conséquent, la rencontre du visiteur avec le musée de niche n'est pas fortuite. Il choisit s'il décide de visiter un tel espace ou non, mais ces rencontres sont le résultat d'un goût formé au fil du temps et qui s'inscrit dans un contexte. Par exemple, si un Musée du Parfum ne donne pas envie de le visiter, ce fait n'est pas dû à la personne, mais aussi à une mentalité collective, sociale. Le parfum est un sujet mineur et marginal, même frivole et certainement sans importance dans certaines cultures. Et pourtant, dans ce contexte, en dépit de ces stéréotypes, il y a aussi des gens curieux, la curiosité étant une autre caractéristique, cette fois une caractéristique du visiteur « niché », un visiteur qui par simple curiosité peut parcourir ce musée.

Pour des chercheurs comme Anne Raulin, la ville est un " théâtre urbain », terme utilisé par l'auteur Anne Raulin pour désigner la ville comme un espace de représentation, parce qu'il abrite la scène de tous les moyens de représentation d'une communauté. Pour le musée de niche, la ville n'est que l'outil d'accès dans ces musées qui change la perception de la visite au musée et de la manière d'évaluation d'une politique culturelle de succès.

Corina Nicolescu, dans Muzeologie Generală, appelle les visiteurs du musée des « amoureux des vieux trucs » (Nicolescu, 1979: 14), mais à l'époque contemporaine le musée n'est plus un dépositaire des objets antiques, il se réinvente d'une culture à une autre, d'une période à une autre. Le visiteur est réinventé en même temps que musée. Il n'est pas par hasard qu'ICOM veut modifier la définition du musée (Sandahl, 2017). Les changements sociaux attirent des modifications culturelles. Il devient inévitable de changer la définition du musée, comme il est aussi inévitable de changer les politiques culturelles. Les contextes culturels sont ceux qui 
définissent la pratique symbolique et, même si elle a un poids considérable, le rôle du musée change, se développe, se modifie en ajoutant des fonctions nouvelles.

Souvent, le musée de niche expose le patrimoine culturel immatériel, c'est-à-dire le savoir-faire, le métier. Voici pourquoi, le musée a dans sa composition, à part le rôle éthique, le rôle éducatif, le rôle de préservation et le rôle de sauvegarde. La plus rapide, pratique et accessible forme de sauvegarde du patrimoine est la création d'un projet culturel. Soit que l'on parle d'une galerie d'art ou d'un musée, la dimension prend de l'importance en nous référant à sa caractéristique principale - l'objet mis en patrimoine. Les aspects du processus de sauvegarde du patrimoine se trouvent aussi en liaison étroite avec le type de patrimoine. Nous pouvons parler du patrimoine culturel matériel, du patrimoine culturel immatériel, du petit patrimoine, du patrimoine industriel, du grand patrimoine ou de ce que André Chastel appelait « la petite cuillère ». Ainsi, dans le processus de patrimonialisation, il y a des objets que nous croyions importants, qui font partie de notre vie quotidienne, que nous utilisons habituellement, mais qui ne font pas l'objet de la création d'un musée du siècle dernier. Par exemple, un musée pour la vaisselle. En général, ces objets font partie d'un projet plus ample envisageant plusieurs catégories (Musée des Arts Décoratifs). Celle-ci ne représente pas un thème indépendant afin de pouvoir se transformer dans un musée autonome.

Même si le but principal d'un musée est d'amener le visiteur dedans, son développement social, culturel et économique ne passe pas au travers. Il est connu le fait qu'un musée ne contribue pas seulement au développement de la culture de la ville, mais à tout son spectre culturel. Voici pourquoi, à présent un musée n'est plus seulement un établissement qui abrite des objets qui font partie du patrimoine, mais il devient un établissement où le dialogue ne s'arrête jamais.

Le but d'un musée, n'importe sa valeur et sa dimension, est de transmettre un message, soit de nature philosophique, esthétique, politique, morale, historique ou scientifique. En allant plus loin, à part la fonction symbolique accomplie, presque chaque musée a un but précis : partager tout ce qui peut être transmis dans une seule visite. Du point de vue éducationnel, il faut réaliser cela le plus possible, parce que l'on ne peut pas anticiper quel type de visiteur existe à un moment donné ou à un autre (Mills, 1955 : 1004). En plus, on ne connait pas son bagage culturel ou éducationnel, ce qui représente son unicité. Un travail d'équipe préparé d'une manière professionnelle longtemps à l'avance a comme objectif de capter toute lattention du visiteur.

Si nous faisons référence au nombre total de musées existants sur le territoire d'un pays nous pouvons comparer la France (Ministère de la 
Culture), avec ses 1219 musées et l'Italie, qui a fait développer le nombre de ses musées en 2011 en arrivant à 3847 ouverts au public (INS, 2013). Le nombre élevé des musées ne signifie pas nécessairement une politique culturelle correcte mais le fait que les acteurs impliqués sont déterminés à accorder au musée la place appropriée. La culture italienne a offert aux sociétés le musée de l'Histoire et le musée de l'Antiquité, tels qu'ils sont connus de nos jours (Nicolescu, $1979: 21$ ). Les transformations du musée se trouvent dans un lien direct avec les transformations culturelles. Ce que 1'État italien offre au musée est un espace d'exposition, tandis que la culture française est celle qui s'intéresse à l'élément social du musée. Comment exposons-nous ? Pourquoi ? Comment amenons-nous le visiteur au musée ? Qu'est-ce que nous devons y exposer? La fusion des deux influences majeures a réussi à amener dans l'époque postmoderne le musée d'aujourd'hui. En apportant en discussion des problèmes d'ordre législatif, une courte analyse comparative a été nécessaire avec un autre espace européen, espace qui met l'accent sur l'histoire du musée et son développement. La preuve est faite par plus de 3000 musées italiens. Dans une courte analyse comparative avec l'espace roumain, nous constatons que dans la culture urbaine italienne existe une identité régionale et locale assumée par l'exposition par la mise en valeur des différents objets par l'intermédiaire des musées. Dans ce cas, pour l'espace roumain une priorité serait de créer le milieu législatif propice pour un tel développement.

Après la Révolution française, l'état français a créé un espace pour rassembler tous les œuvres d'art de l'ancienne monarchie. Cela a également été fait pour avoir un meilleur inventaire de ce qui restait dans le patrimoine national. Il a attribué au musée une importance majeure qui a subi par la suite des modifications de forme et de fond, en acquérant une importance et une exemplarité mondiale. Selon les dernières publications, le musée le plus connu de la France, le Louvre, a enregistré une croissance de visiteurs en 2018, de 10,2 millions de visiteurs (Le Figaro, 2019), la plus grande croissance de tous les temps. Par rapport au Complexe Muséal d'Auschwitz, par exemple, qui a enregistré en 2018 2,1 millions de visiteurs, selon le site officiel (Musée d’Auschwitz-Birkenau, 2018), le Louvre devient le musée le plus visité à l'heure actuelle, enregistrant des chiffres record. Avec un tel pouvoir d'image, le musée français a la possibilité, la capacité et le devoir e transmettre des informations attractives au public, et l'accès à toute forme d'art et à tout acte artistique est fait sans barrières.

D'autres états se sont inspirés du modèle français et du modèle italien. Aussi des initiatives différentes qui approchent le public du musée, donc de la culture régionale et locale, les deux étant essentielles pour l'espace culturel et les politiques culturelles. 
Nous ne pouvons pas parler du musée et de son apport si nous n'amenons pas en discussion une invention qui a connu un réel développement les dernières années : les applications sur le portable. Une attention particulière doit être accordée aux applications du type Google Maps qui sont importantes parce que chaque visiteur les utilise pour calculer les distances, le trafic, les adresses, l'agglomération etc. C'est la raison pour laquelle cette application est utilisée mensuellement par plus de deux milliards de personnes (Popper, 2017), en devenant ainsi l'application des téléphones intelligents la plus utilisée (Panko, 2018). Comme ça, avec un pourcentage si important, les applications comme Google Maps deviennent des outils de recherche pour les experts. Les applications sont très exactes et les utilisateurs peuvent profiter toujours des dernières données grâce aux satellites. En ce qui concerne l'importance accordée par cette application aux musées, nous pouvons observer l'emplacement sur la carte des repères importants par une simple recherche.

Tels que les guides dont on vient de parler, les applications de ce genre s'avèrent un accompagnement virtuel, et remplaceront les guides classiques. Il s'agit aussi ici de l'évolution de la technologie qui, inévitablement, accapare cet espace. En plus, pour pouvoir profiter des deux, les guides classiques comme Michelin Travel Guide sont maintenant offerts sous forme d'application aussi.

En parlant du musée, on parle aussi de la liaison avec la société du visiteur - en lien direct avec l'offre du marché. Pierre Bourdieu fait la distinction entre deux grandes catégories : la production pure et la production de masse. Pour pouvoir parler de l'existence du musée de niche il faut retourner à la demande et à l'offre du marché muséographique.

« La production pure a comme destination un marché limité, tandis que la production de masse est orientée vers la satisfaction des besoins du grand public » (Bourdieu, 2007 : 170). Le musée de niche peut être inscrit dans la catégorie de la production pure, parce que le marché limité peut être comparé à un public limité dans un certain domaine. Prenons l'exemple d'un musée d'anthropologie. Un musée d'anthropologie est destiné premièrement à ceux qui sont intéressés par ce sujet, dans le sens où il abrite tout un système qui ne présente aucun intérêt pour le grand public. D'autre part, un tel musée thématique peut être visité par toute personne qui a la curiosité de le faire, mais la satisfaction des besoins dans un tel cas touche clairement un public restreint. La grande production, comme par exemple les musées nationaux, touche toutes les catégories sociales et professionnelles de tout âge.

Derrière toutes ces données se trouve un discours spécifique à chacun, discours positionné en antithèse avec ce que le musée représente en soi. Les données présentées dans l'analyse de discours de la direction du musée sont les mêmes avec la définition du musée ? Quel est le niveau de similitude 
entre ce que l'on dit et ce qui existe ? Ainsi, nous avons analysé 5 cas de musées de villes européennes différentes, dans l'intervalle 2016-2018 : Le Musée du Parfum de Bucarest, le Musée du Parfum de Barcelone, le Musée Interactif Leonardo Da Vinci de Milan, le Musée des Diamants d'Amsterdam et le Musée Flamenco - Malaga.

Lorsque nous parlons des pratiques du musée, nous parlons de la totalité des besoins assouvis par un musée lorsque nous nous rapportons à son rôle social.

Compte tenu des fonctions d'un musée - médiation, esthétique, educative, conservation et sauvegarde - , nous pouvons parler de plusieurs types d'expériences consommées dans un musée.

A tout cela s'ajoute la fonction symbolique, qui prend contour en produisant les émotions positives et négatives. L'étude de Nathalie Heinich, Les émotions patrimoniales : de l'affect à l'axiologie, représente le point de départ dans l'analyse de la fonction symbolique.

Les observations concernant la fonction symbolique du musée de niche sont présentées par rapport à cinq perspectives :

1. l'évolution du musée (pourquoi?) ;

2. l'implication des autorités locales ;

3. le message du musée ;

4. l'importance du musée ;

5. l'empreinte sociale du musée.

En tenant compte des informations qui sont parvenues suite à l'analyse de discours, l'accès dans ces musées est venu comme une confirmation ou une infirmation de ce qui a été écrit. Par le biais de l'observation non participative nous avons cherché à observer et à analyser le musée en soi. Mais, le but des visites a été la recherche sur ce que l'on dit et sur ce qu'il existe.

Les cinq perspectives suivies durant la visite des musées ont montré le fait que nous ne pouvons pas parler d'une pratique symbolique si les fonctions élémentaires du musée ne sont pas remplies. Ceci devient un symbole de la société où il s'inscrit seulement s'il remplit ses autres fonctions. Ainsi, la patrimonialisation du métier, c'est-à-dire du savoir-faire, dans le postmodernisme devient possible en connaissant les habiletés de l'autrui. C'est à dire que le savoir-faire n'atteint pas ses pleins pouvoirs s'il n'y a pas une maîtrise complète des connaissances de tout ce que le domaine étudié implique. Inévitablement, une connaissance de l'autrui « conduira à une meilleure connaissance de soi » (Laplatine, 2000 : 17).

Dans la perspective des cinq dimensions, nous avons pu énoncer les observations suivantes : le musée de niche s'inscrit dans un cycle évolutif de la société, c'est-à-dire au fur et à mesure que la société évolue ou change, il le fait aussi. En même temps, l'aspect privé du musée de niche s'est transformé 
dans une condition pour qu'il fasse partie de la présente catégorie. Il n'est pas par hasard que des personnes privées, des fondations privées ou même des familles gèrent ces musées. En même temps, «la niche» se réfère à un confinement de l'objet mis en patrimoine, et non pas à la dimension. Finalement, tel que l'on l'observera, le musée de niche est un musée du patrimoine culturel intangible défini dans le cadre de la Convention UNESCO de Paris (Convention UNESCO, 2003).

Dans l'analyse des musées nous pouvons tracer une série d'idées particulières à chacun :

Le Musée du Parfum de Barcelone : « un objet n'a pas de sens s'il n'est pas lié au contexte particulier dans lequel s'inscrit » (Deliege, 2007 :126), tel que ce contexte particulier est l'histoire du parfum, l'histoire de Barcelone et, in extenso, une petite partie de l'histoire de l'Espagne. Le fait que le parfum est un symbole de la richesse démontre du point de vue historique, géographique et sociologique que l'Espagne, une ex-monarchie, a joui du fait qu'une partie de la population qui investissait dans un tel objet appréciait et comprenait son sens. En même temps, le même contexte nous a montré un besoin dans la société, du point de vue culturel, d'une histoire écrite, olfactive et visuelle du parfum. Il a été nécessaire d'apporter dans le processus de mise en patrimoine un petit flacon plein avec des essences réalisées par un processus chimique.

Le Musée du Parfum de Bucarest : Le Musée du Parfum de Bucarest est un musée de niche qui met en patrimoine l'objet cosmétique prédominant du XXIème siècle (Labut, 2013). Il devient un symbole du processus de patrimonialisation grâce au savoir-faire détenu par chaque flacon. Autrement dit, chaque musée s'inscrit dans un certain contexte social. Ce cas-ci, le Musée du Parfum de Bucarest présente une collection de parfums disparus qui ont fait partie de la production régionale de l'école de parfumeurs. Tout en sachant qu'en Roumanie il y a eu ce type de culture. Le musée de niche maintient vif le souvenir, l'image ou le flacon de parfum, objet représentatif pour la periode de l'entre-deux-guerres.

Dans une ville comme Milan, ville cosmopolite, Le Musée Interactif Leonardo da Vinci se trouve dans la liste des grands musées abrités par la ville. L'Italie est connue comme le pays avec le plus grand nombre de musées. Tous importants, parce que les Italiens ont compris la manière de promouvoir la culture et de réaliser une introspection dans leur culture. $\mathrm{Ce}$ musée est interactif. Il n'est pas un simple musée qui expose une série d'objets mais il invite le consommateur culturel (le visiteur) à créer lui-même. Pour cette raison, le musée jouit d'une popularité élevée parmi les touristes, fait qui ressort des médias en ligne. Ce musée de niche est un bon exemple pour la manière de réaliser un musée qui ait une composante éducationnelle 
mais aussi une partie nichée par le sujet (les inventions) et non pas par celui qui a produit lesdites inventions (Leonardo Da Vinci).

Le Musée des Diamants d'Amsterdam : Il s'agit d'un musée qui n'a pas de grandes dimensions, mais un musée qui jouit d'une notoriété exquise. Son fondateur a démarré ce projet par passion, seulement pour mettre en patrimoine et pour transmettre plus loin le métier de gemmologue et de bijoutier en même temps. L idée est de créer un espace qui puisse permettre l'explication et la pratique du métier, métier qui a caractérisé une partie considérable de la population d'Amsterdam, métier qui fait partie de l'histoire du Pays-Bas. C'est le but de l'UNESCO, de l'ICOM et d'autres organisation de garder et encourager un métier comme celui-ci-dessus. Ce que l'on met dans le patrimoine sont la connaissance et l'habileté de produire un métier traditionnel (UNESCO), afin d'éduquer les générations suivantes et de promouvoir la créativité humaine. Ce métier fait partie du patrimoine (Niţulescu, 2008 :3) néerlandais et il doit être traité comme tel par les intervenants culturels et politiques. Traité de cette manière, il devient un « testament extrêmement convaincant, et la manière de le lire signifie une meilleure compréhension de la société où on vit » (Hedeşan, $2008: 3$ ).

Ce musée est un réel exemple pour le musée de niche. En moyenne, selon les données fournies par le site officiel, il y a plus de 750.000 visiteurs chaque année, ce qui signifie une popularité croissante parmi les visiteurs pour ce genre de musée.

« L'œuvre d'art, en tant qu'avoir symbolique (et non pas économique, même s'il peut l'être aussi), ne peut exister comme tel que pour une personne qui a les moyens nécessaires pour y accéder ou, autrement dit, pour le déchiffrer. Le niveau de compétence artistique d'un individu est donné par le degré où il maîtrise à un moment donné le kit d'outils nécessaires à la compréhension de l'œuvre d'art, c'est-à-dire les schémas d'interprétation sans lesquels il ne peut pas s'approprier le capital artistique $[\ldots]$ si ces conditions particulières ne sont pas remplies, la compréhension erronée est inévitable : l'illusion d'une compréhension immédiate conduit à une perception illusoire, fondée sur un code erroné » (Mihăilescu, 2007:39).

En tenant compte de ce que Vintilă Mihăilescu à chaque visiteur revient la mission d'interpréter ce qu'il voit de sa perspective de compréhension de l'œuvre d'art. Voici pourquoi la naissance d'un musée est difficile. Il ne s'agit pas de la partie législative, mais de la manière dont l'on introduit le visiteur dans son histoire.

Le Musée Flamenco de Malaga : Selon Chiara Bortolotto, les notionsclés du patrimoine culturel immatériel sont la culture, l'identité, la tradition (Bortolotto, 2011 :24), aspects retrouvés dans le moindre détail au sein de ce 
musée. Dans ce cas, la nouveauté n'est pas le fait que le musée mettait en patrimoine le flamenco, mais la manière de définir ses objectives, grâce à laquelle le Musée Flamenco de Malaga mérite d'être mentionné pour les politiques culturelles activées. En outre, Chiara Bortolotto souligne la capacité du patrimoine culturel immatériel d'être reproduit, chose réalisée dans le musée. Il est évident que le musée de niche devient l'un du patrimoine culturel immatériel dans ce cas-ci, caractéristique de cette catégorie de musées. Le Flamenco a pris ampleur en devenant non seulement un spécifique local, mais aussi global. Ainsi la tentative du musée d'être en même temps local et global est accomplie même au cœur de ce petit musée. Il devient important pour le spécifique local, mais il devient connu globalement justement par la transmission de cet élément de patrimoine. L'inscription sur la liste de l'UNESCO a aidé d'une manière significative par le fait que l'accès du public large à la création artistique est fait plus facilement. En créant un musée du spécifique local, on crée un espace de rappel des artistes, des auteurs et des chansons qui gardent vif l'esprit du flamenco.

Le Musée du Fado de Lisbonne : La musique fado devient un repère d'identité et d'identification des Portugais dans l'espace européen. Dans les programmes locaux et nationaux, le musée est mentionné comme partie du produit des acteurs locaux impliqués ${ }^{2}$. L'implication des autorités locales dans le démarrage d'un musée de niche est limitée. En général, les musées de niche naissent d'une passion commune d'un groupe d'une communauté. L'implication des autorités peut intervenir seulement dans une éventuelle promotion locale et régionale, mais non à la création du musée. Cette raison est due aux fondateurs du musée de niche, qui, conduits par une passion, réussissent à créer un espace de remémoration. L'intervention des autorités locales ne produirait qu'une diminution de la création et de l'originalité. Cette implication pourrait être seulement financière, mais ne relèverait pas de la direction stratégique culturelle.

Nous avons utilisé trois méthodes d'analyse (la méthode comparative, l'analyse de discours et l'observation non participative) en étroite liaison avec l'analyse des documents primaires (Loi 311/2003 - Roumanie, Loi 20025/2002 - France, Decreto Musei - Italie). La législation du musée dans ces trois pays reste incertaine en ce qui concerne le musée de niche même si son existence est attestée par tous ces musées analysés. En même temps, les trois méthodes d'analyse se sont trouvées en liaison étroite avec cinq perspectives

\footnotetext{
${ }^{2}$ With this increase and renovation of the exhibition circuit, the Museu do Fado earned, in 2009, several prizes and awards including the Prize Essay and Disclosure from the Amália Rodrigues Foundation, the Honorable Mention - Best Portuguese Museum from APOM (Portuguese Association of Museology), and the classification, by Tourism of Portugal, among the five finalists in the category of "Public Rehabilitation Project", extrait du site officiel du musée.
} 
d'analyse : l'évolution du musée, l'implication des autorités locales, le message du musée, l'importance du musée, l'empreinte sociale du musée.

L'initiative ICOM de 2018 n'est pas fortuite et elle propose une redéfinition mise à jour du musée, en concordance avec tous les changements qui se sont passés dans le monde. En même temps, chaque personne est invitée à inscrire une définition considérée appropriée comme la définitioncanon du musée (ICOM, 2017). Son enregistrement peut se faire sur le site officiel d'ICOM et ensuite les représentants de l'établissement choisiront la plus appropriée et adaptée définition du musée (ICOM, MDPP, 2019).

Au mois d'août 2019, ICOM a choisi une définition générale du musée pour être soumise au vote :

« Museums are democratising, inclusive and polyphonic spaces for critical dialogue about the pasts and the futures. Acknowledging and addressing the conflicts and challenges of the present, they hold artefacts and specimens in trust for society, safeguard diverse memories for future generations and guarantee equal rights and equal access to heritage for all people.

Museums are not for profit. They are participatory and transparent, and work in active partnership with and for diverse communities to collect, preserve, research, interpret, exhibit, and enhance understandings of the world, aiming to contribute to human dignity and social justice, global equality and planetary wellbeing » (ICOM, 2019).

Par suite, nous assistons à un changement en simultané avec le changement social politique global.

Dans son livre L'Anthropologie, à quoi bon?, Georges GuillesEscuret affirme que : "L`anthropologie ne sert à rien. Qui peut prétendre aujourd hui le contraire et au nom de quoi ? »(Guilles-Escuret, $1996: 7$ ). Une réponse possible peut résider dans l'analyse des musées de niche qui nous prouve que les arts et les sciences humaines réussissent à fusionner dans un superbe projet intitulé le musée de niche.

\section{Références :}

Bortolotto, C. (2011). Le trouble du patrimoine culturel immatériel/ The problem with intangible cultural heritage. In Le patrimoine culturel immatériel, Paris, Éditions de la Maison des sciences de l'homme. https://doi.org/10.4000/gradhiva.2412.

Bourdieu, P. (2007). Regulile artei/The rules of art. Bucureşti: Editions ART.

Deliège, R. (2007). O istorie a antropologiei/ A history of anthropology. Bucureşti: Cartier. 
Guilles-Escuret, G. (1996). L'anthropology, à quoi bon? Chercheurs, techniciens, intellectuels et militants/ Anthropology, for what is good? Researchers, technicians, intellectuals and militants. Paris : L`Harmattan.

Hedeşan, O. (2008). The Mărţişor. Or Reinventing a Tradition. Baia Mare : Editura Universităţii de Nord

Heinich, N. (2009). La fabrique du patrimoine - De la cathédrale à la petite cuillère. Paris: Maison des Sciences de l'homme

Kent, H. (1913). The Small Museum. In Art and Progress, 4(10), 1048.

Laplantine, F. (2000). Descrierea etnograficăl Ethnographic description. Iaşi : Polirom.

Matei Călinescu, M. (2017). Cinci fețe ale modernităţii/ Five faces of modernism. Iaşi: Polirom.

Mihăilescu, V. (2009). Antropologie. Cinci introduceril Anthropology. Five introductions. Iaşi: Polirom.

Mills, G. (1955). Social Anthropology and the Art Museum. In American Anthropologist, New Series, 57(5), 1004-1005. https://doi.org/10.1525/aa.1955.57.5.02a00070

Nicolescu, C. (1979). Muzeologie generală/ General museography. Bucureşti: Editura Didactică şi Pedagogică

Niţulescu, V. et.alt. (2008). Repertoriul naţional de patrimoniu cultural imateriall National repertory of intangible cultural heritage. In CIMEC, Volume I, Bucureşti.

\section{Ressources électroniques :}

ICOM. (2017). The challenge of revising the museum definition, disponible à l'adresse suivante: https://icom.museum/en/news/the-challenge-of-revisingthe-museum-definition/, accédé le 19.10.2020

Standing Committee for Museum Definition, Prospects and Potentials. (2019). In ICOM Conference, disponible à l'adresse suivante: https://icom.museum/wp-content/uploads/2019/01/MDPP-report-andrecommendations-adopted-by-the-ICOM-EB-December-2018 EN-2.pdf, accédé le 19.10.2020

ICOM. (2019). ICOM announces the alternative museum definition that will be subject to a vote, disponible à l'adresse suivante: https://icom.museum/en/news/icom-announces-the-alternative-museumdefinition-that-will-be-subject-to-a vote/?fbclid=IwAR16jWqlWoYLWv82Rm2m89u N9ewhezDUems1LZa1caWxfl6wr_07e_MRQ, accédé le 19.10.2020

Instituto Nazionale di Statistica. (2013). Museums, archeological areas and monuments in Italy, disponible à l'adresse suivante: https://www.istat.it/en/archive/106183, accédé le 19.10.2020

Ministère de la Culture, Appellation "Musée de France », disponible à l'adresse suivante: http://www.les-musees-de-france.fr/, accédé le 19.10.2020 
Labut C. (2013). Neuf françaises sur dix se parfument, disponible à l’adresse suivante : https://www.lexpress.fr/styles/parfums/neuf-francaises-sur-dix-separfument_1261781.htm, accédé le 19.10.2020

Le Figaro (2019). Avec 10,2 millions de visiteurs, le Louvre a battu un nouveau record de fréquentation en 2018, disponible à l'adresse suivante : http://www.lefigaro.fr/culture/2019/01/03/03004-20190103ARTFIG00071avec-102-millions-de-visiteurs-le-louvre-a-battu-un-nouveau-record-defrequentation-en-2018.php, accédé le 19.10.2020

Loi $2002 / 5$ du 04.01.2002, disponible à l'adresse suivante:https://www.legifrance.gouv.fr/affichTexte.do?cidTexte=JORFTE XT000000769536\&categorieLien=id, accédé le 19.10.2020

Loi des Musées de l'Italie, disponible à l'adresse suivante : http://www.beniculturali.it/mibac/export/MiBAC/sito MiBAC/Contenuti/Ministero/visualizza_asset.html_1947521712.html, accédé le 19.10.2020

Musée d'Auschwitz-Birkenau (2018). 2,1 million visitors at the Memorial, disponible à l'adresse suivante: http://auschwitz.org/en/museum/news/2-1million-visitors-at-the-memorial-in-2017,1292.html, accédé le 19.10.2020

Panko. R. (2018). The popularity of Google Maps: Trends in Navigation Apps, disponible à l'adresse suivante: https://themanifest.com/appdevelopment/popularity-google-maps-trends-navigation-apps-2018, accédé le 19.10 .2020

Popper. B. (2017). Google announces over 2 billion monthly active devices on Android, disponible à l'adresse suivante : https://www.theverge.com/2017/5/17/15654454/android-reaches-2-billionmonthly-active-users, accédé le 19.10.2020

UNESCO, What is Intangible Cultural Heritage?, disponible à l'adresse suivante: https://ich.unesco.org/en/what-is-intangible-heritage-00003 accédé le $\underline{19.10 .2020}$ 